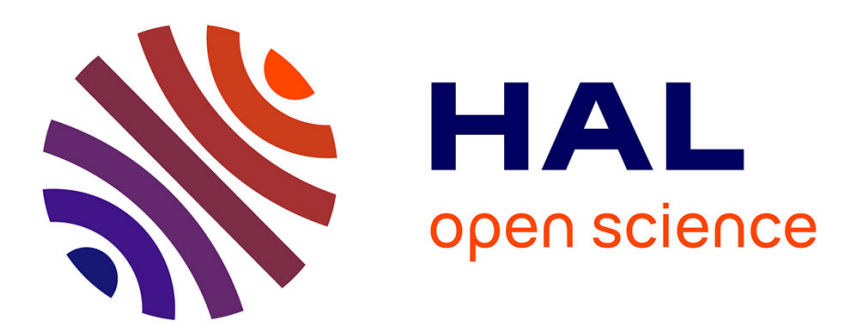

\title{
Variational linear comparison bounds for nonlinear composites with anisotropic phases. I. General results
}

\author{
Martin I Idiart, Pedro Ponte Castañeda
}

\section{To cite this version:}

Martin I Idiart, Pedro Ponte Castañeda. Variational linear comparison bounds for nonlinear composites with anisotropic phases. I. General results. Proceedings of the Royal Society A: Mathematical, Physical and Engineering Sciences, 2007, 463, pp.907-924. 10.1098/rspa.2006.1797 . hal-00311889

\section{HAL Id: hal-00311889 \\ https://hal.science/hal-00311889}

Submitted on 28 Aug 2018

HAL is a multi-disciplinary open access archive for the deposit and dissemination of scientific research documents, whether they are published or not. The documents may come from teaching and research institutions in France or abroad, or from public or private research centers.
L'archive ouverte pluridisciplinaire HAL, est destinée au dépôt et à la diffusion de documents scientifiques de niveau recherche, publiés ou non, émanant des établissements d'enseignement et de recherche français ou étrangers, des laboratoires publics ou privés. 


\title{
Variational linear comparison bounds for nonlinear composites with anisotropic phases. I. General results
}

\author{
By Martín I. Idiart ${ }^{1,2}$ and Pedro Ponte Castañeda ${ }^{1,2, *}$ \\ ${ }^{1}$ Department of Mechanical Engineering and Applied Mechanics, \\ University of Pennsylvania, Philadelphia, PA 19104-6315, USA \\ ${ }^{2}$ Laboratoire de Mécanique des Solides, C.N.R.S. UMR 7649, \\ Département de Mécanique, École Polytechnique, \\ 91128 Palaiseau Cedex, France
}

\begin{abstract}
This work is concerned with the development of bounds for nonlinear composites with anisotropic phases by means of an appropriate generalization of the 'linear comparison' variational method, introduced by Ponte Castañeda for composites with isotropic phases. The bounds can be expressed in terms of a convex (concave) optimization problem, requiring the computation of certain 'error' functions that, in turn, depend on the solution of a non-concave/non-convex optimization problem. A simple formula is derived for the overall stress-strain relation of the composite associated with the bound, and special, simpler forms are provided for power-law materials, as well as for ideally plastic materials, where the computation of the error functions simplifies dramatically. As will be seen in part II of this work in the specific context of composites with crystalline phases (e.g. polycrystals), the new bounds have the capability of improving on earlier bounds, such as the ones proposed by deBotton and Ponte Castañeda for these specific material systems.
\end{abstract}

Keywords: nonlinear homogenization; variational methods; anisotropy

\section{Introduction}

For a linear-elastic constitutive response, there are well-established methods to estimate the effective or overall behaviour of composite materials. These so-called homogenization methods include the variational principles of Hashin \& Shtrikman (1963), which can be used to bound the effective modulus tensor of linear-elastic composites. A comprehensive review of this and other works on linear composites is given, for example, in the recent monograph by Milton (2002).

On the other hand, for nonlinear (e.g. plastic, viscoplastic, etc.) composites, rigorous methods have not been available until more recently, even though efforts along these lines have been going on for some time, particularly in the context of ductile polycrystals (e.g. Hill 1965; Hutchinson 1976). Making use of a nonlinear extension of the Hashin-Shtrikman (HS) variational principles, due to Willis (1983), the first bounds of the HS type for nonlinear composites were

* Author for correspondence (ponte@seas.upenn.edu). 
derived by Talbot \& Willis (1985). Further applications of this methodology to composites with nonlinear anisotropic phases (e.g. polycrystals) were given by Willis (1994). A more general variational approach making use of the notion of optimally chosen 'linear comparison composites' was proposed by Ponte Castañeda (1991). This approach is not only capable of delivering bounds of the HS type for nonlinear composites, but also, in addition, can be used to generate bounds and estimates of other types, such as self-consistent estimates and three-point bounds (Ponte Castañeda 1992). The connections between these two different approaches were explored by Willis (1992) and Talbot \& Willis (1992). Suquet (1993) and Olson (1994) proposed alternative, but equivalent methods for the special classes of power-law and ideally plastic materials, respectively. Suquet (1995) also gave a novel physical interpretation of the variational estimates of Ponte Castañeda (1991) in terms of 'secant' moduli and the second moments of the local fields in the linear comparison composite. In addition, deBotton \& Ponte Castañeda (1995) proposed an extension of the variational principle of Ponte Castañeda (1991) to generate bounds for composite materials with (anisotropic) viscoplastic crystalline phases. This variational approach was, in turn, generalized further by Suquet (see Ponte Castañeda \& Suquet 1998) to include more general types of anisotropies in the context of nonlinear composites. Finally, it should be mentioned that there is another approach, called the 'translation' method, which has been applied successfully to polycrystals, but thus far only for scalar potential problems (Kohn \& Little 1998; Nesi et al. 2000; Goldsztein 2001; Garroni \& Kohn 2003).

The present work is concerned with an extension of the variational method of Ponte Castañeda (1991, 1992) for composites with nonlinear (viscoplastic) anisotropic phases. It will be shown that this generalization, which is more closely tied to the original formulation, has the capability to give improved bounds relative to the generalizations provided by deBotton \& Ponte Castañeda (1995) in the specific context of viscoplastic polycrystals, and the further generalization provided by Ponte Castañeda \& Suquet (1998) for more general anisotropies.

Thus, attention will be focused on composite materials made of $N$ homogeneous constituents, or phases, that are randomly distributed in a specimen occupying a volume $\Omega$ at a length-scale which is much smaller than the size of the specimen and the scale of variation of the loading conditions. The constitutive behaviour of the nonlinear (viscoplastic) anisotropic phases is characterized by convex strain (strain-rate) potentials $w^{(r)}(r=1, \ldots, N)$, satisfying the conditions $w^{(r)}(\boldsymbol{O})=0$ and $w^{(r)}(\boldsymbol{\varepsilon}) \rightarrow \infty$ as $|\boldsymbol{\varepsilon}| \rightarrow \infty$. The microstructure is defined by characteristic functions $\chi^{(r)}$ that are equal to 1 if the position vector $\boldsymbol{x}$ is in phase $r$ (i.e. $\boldsymbol{x} \in \Omega^{(r)}$ ), and 0 otherwise. In this case, the relation between the stress $\boldsymbol{\sigma}$ and the strain (strain-rate) $\boldsymbol{\varepsilon}$ is defined by a local strain potential $w$, such that

$$
\boldsymbol{\sigma}=\frac{\partial w}{\partial \boldsymbol{\varepsilon}}(\boldsymbol{x}, \boldsymbol{\varepsilon}), \quad w(\boldsymbol{x}, \boldsymbol{\varepsilon})=\sum_{r=1}^{N} \chi^{(r)}(\boldsymbol{x}) w^{(r)}(\boldsymbol{\varepsilon}) .
$$

The problem is then that of determining the effective behaviour of the composite, which is defined as the relation between the average stress $\overline{\boldsymbol{\sigma}}=\langle\boldsymbol{\sigma}\rangle$ and the average strain $\bar{\varepsilon}=\langle\boldsymbol{\varepsilon}\rangle$. Here, the symbols $\langle\cdot\rangle$ and $\langle\cdot\rangle^{(r)}$ will be used to denote volume averages over the composite $(\Omega)$ and phase $r\left(\Omega^{(r)}\right)$, respectively. For the class of materials characterized by relations (1.1), the effective behaviour 
is determined by the effective strain potential, defined by

$$
\tilde{w}(\overline{\boldsymbol{\varepsilon}})=\inf _{\boldsymbol{\varepsilon} \in \mathcal{K}(\overline{\boldsymbol{\varepsilon}})}\langle w(\boldsymbol{x}, \boldsymbol{\varepsilon})\rangle=\inf _{\boldsymbol{\varepsilon} \in \mathcal{K}(\overline{\boldsymbol{\varepsilon}})} \sum_{r=1}^{N} c^{(r)}\left\langle w^{(r)}(\boldsymbol{\varepsilon})\right\rangle^{(r)},
$$

where $c^{(r)}=\left\langle\chi^{(r)}\right\rangle$ denotes the volume fraction of phase $r$; and $\mathcal{K}(\overline{\boldsymbol{\varepsilon}})$ is the set of 'kinematically admissible' strain fields, such that there is a continuous displacement field $\boldsymbol{u}$ satisfying $\boldsymbol{\varepsilon}=(1 / 2)\left[\nabla \boldsymbol{u}+(\nabla \boldsymbol{u})^{\mathrm{T}}\right]$ in $\Omega$, and $\boldsymbol{u}=\overline{\boldsymbol{\varepsilon}} \boldsymbol{x}$ in $\partial \Omega$. It is important to recall that (strict) convexity of the local strain potential $w$ on the local strain $\varepsilon$ implies (strict) convexity of the effective potential $\tilde{w}$ in the applied strain $\bar{\varepsilon}$.

The effective stress-strain relation is then known to be given by

$$
\overline{\boldsymbol{\sigma}}=\frac{\partial \tilde{w}}{\partial \bar{\varepsilon}}(\overline{\boldsymbol{\varepsilon}}),
$$

where it has been assumed that $\tilde{w}$ is differentiable, as has been done for the local potential $w$ in expression (1.1). In practice, the local and effective potentials can be assumed to be differentiable for most material models of interest, except in some special cases, including the ideally plastic materials to be considered further in $\S 4 b$, where the potential is not differentiable (everywhere), but is still convex (although not strictly so). In this case, it is most natural to work with the subdifferential of convex analysis (Ekeland \& Temam 1999). Here, for simplicity, the distinction will not be made between standard (Gateaux) derivatives and subdifferentials, except when it becomes necessary or convenient to do so.

Alternatively, the behaviour of phase $r$ may be characterized by a convex stress potential $u^{(r)}$, defined by the Legendre-Fenchel transform, or convex dual (Ekeland \& Temam 1999), of $w^{(r)}$,

$$
u^{(r)}(\boldsymbol{\sigma})=\left(w^{(r)}\right)^{*}(\boldsymbol{\sigma})=\sup _{\boldsymbol{\varepsilon}}\left[\boldsymbol{\sigma} \cdot \boldsymbol{\varepsilon}-w^{(r)}(\boldsymbol{\varepsilon})\right] .
$$

Then, the local stress and strain tensors are related by

$$
\boldsymbol{\varepsilon}=\frac{\partial u}{\partial \boldsymbol{\sigma}}(\boldsymbol{x}, \boldsymbol{\sigma}), \quad u(\boldsymbol{x}, \boldsymbol{\sigma})=\sum_{r=1}^{N} \chi^{(r)}(\boldsymbol{x}) u^{(r)}(\boldsymbol{\sigma}),
$$

and the effective behaviour can be described in terms of the effective stress potential $\tilde{u}$, such that

$$
\overline{\boldsymbol{\varepsilon}}=\frac{\partial \tilde{u}}{\partial \overline{\boldsymbol{\sigma}}}(\overline{\boldsymbol{\sigma}}), \quad \tilde{u}(\overline{\boldsymbol{\sigma}})=\inf _{\boldsymbol{\sigma} \in \mathcal{S}(\overline{\boldsymbol{\sigma}})}\langle u(\boldsymbol{x}, \boldsymbol{\sigma})\rangle=\inf _{\boldsymbol{\sigma} \in \mathcal{S}(\overline{\boldsymbol{\sigma}})} \sum_{r=1}^{N} c^{(r)}\left\langle u^{(r)}(\boldsymbol{\sigma})\right\rangle^{(r)},
$$

where $\mathcal{S}(\overline{\boldsymbol{\sigma}})$ is the set of 'statically admissible' stress fields $\boldsymbol{\sigma}$ that are divergencefree in $\Omega$, satisfying the traction continuity conditions across phase boundaries, and are such that $\langle\boldsymbol{\sigma}\rangle=\overline{\boldsymbol{\sigma}}$. Note that $\tilde{u}$ is convex, and it has also been assumed to be differentiable, for simplicity.

Given the assumed convexity of the phase potentials, the variational formulations (1.2) and (1.6) $)_{2}$ for the effective behaviour of the nonlinear composites can be shown to be completely equivalent, in the sense that the functions $\tilde{w}$ and $\tilde{u}$ are Legendre duals of each other, $\tilde{u}=\tilde{w}^{*}$. Thus, the problem of finding the constitutive relation for the composite reduces to that of determining the effective potentials $\tilde{w}$ or $\tilde{u}$. It is well known (e.g. Hutchinson 1976) that upper and lower bounds for $\tilde{w}$ and $\tilde{u}$ may be obtained by making use of uniform trial 
fields in the context of definitions (1.2) and $(1.6)_{2}$. In the linear case, they are known as the bounds of Voigt and Reuss.

\section{Linear comparison materials}

Consider a linear-elastic comparison material with positive-definite, fully symmetric stiffness tensor $\mathbb{C}_{0}$, such that its strain potential is given by

$$
w_{0}(\boldsymbol{x}, \boldsymbol{\varepsilon})=\frac{1}{2} \boldsymbol{\varepsilon} \cdot \mathbb{C}_{0}(\boldsymbol{x}) \boldsymbol{\varepsilon} .
$$

Assuming that $w^{(r)}$ has 'weaker-than-quadratic' growth at infinity, implying that $\left(w^{(r)}-w_{0}\right) \rightarrow-\infty$ as $|\boldsymbol{\varepsilon}| \rightarrow \infty$ (for fixed $\boldsymbol{x}$ ), suggests the following definition.

Definition 2.1. Let $v^{(r)}$ be the phase 'error' function, serving as a measure of the nonlinearity in the original material with the strain potential $w^{(r)}$, given by

$$
v^{(r)}\left(\mathbb{C}_{0}\right)=\sup _{\varepsilon}\left\{w^{(r)}(\boldsymbol{\varepsilon})-\frac{1}{2} \boldsymbol{\varepsilon} \cdot \mathbb{C}_{0} \boldsymbol{\varepsilon}\right\}
$$

Remark 2.1. Definition (2.2) is still valid when the stiffness tensor $\mathbb{C}_{0}$ is not positive definite, but in this case $v^{(r)}$ takes on infinite values. This is easy to see, because choosing $\varepsilon$ to be proportional to the eigentensor corresponding to any zero, or negative eigenvalue of $\mathbb{C}_{0}$, leads to an infinite value for the supremum in expression $(2.2)$. In fact, $v^{(r)}$ tends to infinity, when any eigenvalue of (a positive definite) $\mathbb{C}_{0}$ tends to zero. Also, note that, by definition, $v^{(r)}\left(\mathbb{C}_{0}\right) \geq w^{(r)}(\mathbf{0})=0$, and hence $v^{(r)}$ is a non-negative function. In addition, being the pointwise supremum of a collection of affine functions of $\mathbb{C}_{0}, v^{(r)}$ is a convex function of $\mathbb{C}_{0}$. Furthermore, assuming smoothness of the functions $w^{(r)}$, the solutions of the non-concave optimization problem defined by (2.2) normally satisfy the stationarity conditions

$$
\mathbb{C}_{0} \hat{\boldsymbol{\varepsilon}}_{(m)}^{(r)}=\frac{\partial w^{(r)}}{\partial \boldsymbol{\varepsilon}}\left(\hat{\boldsymbol{\varepsilon}}_{(m)}^{(r)}\right),
$$

where $\hat{\boldsymbol{\varepsilon}}_{(m)}^{(r)}, m=1, \ldots, M^{(r)}$, denote all the strain tensors for which the maximum is attained in (2.2) for a given $\mathbb{C}_{0}$. (Note that $M^{(r)}$ can be infinite in some instances, such as when the potential $w^{(r)}$ and $\mathbb{C}_{0}$ are both isotropic.)

It follows from definition (2.2) that

for any $\mathbb{C}_{0}$, and hence that

$$
w^{(r)}(\boldsymbol{\varepsilon}) \leq \frac{1}{2} \boldsymbol{\varepsilon} \cdot \mathbb{C}_{0} \boldsymbol{\varepsilon}+v^{(r)}\left(\mathbb{C}_{0}\right),
$$

$$
w^{(r)}(\boldsymbol{\varepsilon}) \leq \inf _{\mathbb{C}_{0}}\left\{\frac{1}{2} \boldsymbol{\varepsilon} \cdot \mathbb{C}_{0} \boldsymbol{\varepsilon}+v^{(r)}\left(\mathbb{C}_{0}\right)\right\} .
$$

But, as has been pointed out earlier, the function $v^{(r)}$ is infinite if $\mathbb{C}_{0}$ is not positive definite, and therefore expression $(2.5)$ can be equivalently written as

$$
w^{(r)}(\boldsymbol{\varepsilon}) \leq \inf _{\mathbb{C}_{0}>0}\left\{\frac{1}{2} \boldsymbol{\varepsilon} \cdot \mathbb{C}_{0} \boldsymbol{\varepsilon}+v^{(r)}\left(\mathbb{C}_{0}\right)\right\}
$$

where the notation $\mathbb{C}_{0}>0$ has been used to signify that $\mathbb{C}_{0}$ is positive definite. In general, the inequality in expression (2.6) will hold. However, there may be classes of nonlinear materials, for which the equality would hold. Noting that the right-hand side of expression (2.6) is concave in the variable $\boldsymbol{\varepsilon} \otimes \boldsymbol{\varepsilon}$ (because it is 
the pointwise infimum of a set of affine functions in this variable) suggests that in order for equality to hold in expression (2.6), the potential $w$ must be concave in the variable $\boldsymbol{\varepsilon} \otimes \boldsymbol{\varepsilon}$. Accordingly, it will be assumed that the potentials $w$ of interest here satisfy the following 'square concavity' hypothesis.

Hypothesis 2.1. It is assumed that there exist functions $f^{(r)}$ that are concave in the space of fourth-order tensors, e, possessing the usual symmetry properties of elasticity tensors, and satisfying the following properties: (i) $f^{(r)}(\mathbb{D})=0$ and (ii) $f^{(r)} \rightarrow \infty$ as $|\mathrm{e}| \rightarrow \infty$, such that the phase potentials $w^{(r)}$ may be expressed as

$$
w^{(r)}(\boldsymbol{\varepsilon})=f^{(r)}\left(\frac{1}{2} \boldsymbol{\varepsilon} \otimes \boldsymbol{\varepsilon}\right) .
$$

This hypothesis was introduced by Ponte Castañeda (1992) for isotropic materials and generalized as described previously for anisotropic materials by Suquet (see Ponte Castañeda \& Suquet 1998). It should be emphasized that the functions $f^{(r)}$ are not uniquely determined. Also, a consequence of hypothesis 2.1 is that the potentials $w^{(r)}$, as defined by relation (2.7), should satisfy the relation $w^{(r)}(-\boldsymbol{\varepsilon})=w^{(r)}(\boldsymbol{\varepsilon})$. In addition, it should be noted that this hypothesis is consistent with the earlier assumption that the potentials $w^{(r)}$ exhibit weaker-than-quadratic growth on the strain $\varepsilon$. It is shown next that the equality holds strictly in expression (2.6) when the stronger square concavity hypothesis is made.

Lemma 2.1. Consider a composite with 'square concave' phase potentials $w^{(r)}$, as defined in hypothesis 2.1. It then follows that

$$
w^{(r)}(\boldsymbol{\varepsilon})=\inf _{\mathbb{C}_{0}>0}\left\{\frac{1}{2} \boldsymbol{\varepsilon} \cdot \mathbb{C}_{0} \boldsymbol{\varepsilon}+v^{(r)}\left(\mathbb{C}_{0}\right)\right\},
$$

where the error functions $v^{(r)}$ have been defined by relations (2.2). It also follows that

$$
w(\boldsymbol{x}, \boldsymbol{\varepsilon})=\inf _{\mathbb{C}_{0}>0}\left\{w_{0}(\boldsymbol{x}, \boldsymbol{\varepsilon})+v\left(\boldsymbol{x}, \mathbb{C}_{0}\right)\right\},
$$

where $w_{0}$ is given by (2.1), and $v$ is the function defined by

$$
v\left(\boldsymbol{x}, \mathbb{C}_{0}\right)=\sup _{\boldsymbol{\varepsilon}}\left\{w(\boldsymbol{x}, \boldsymbol{\varepsilon})-w_{0}(\boldsymbol{x}, \boldsymbol{\varepsilon})\right\} .
$$

Proof. The concave Legendre-Fenchel transform of $f^{(r)}$ is defined as

$$
f_{*}^{(r)}\left(\mathbb{C}_{0}\right)=\inf _{\mathrm{e}}\left\{\mathbb{C}_{0} \cdot \mathrm{e}-f^{(r)}(\mathrm{e})\right\} .
$$

Note that $f_{*}^{(r)}$ is a non-positive, concave function of $\mathbb{C}_{0}$, such that $f_{*}^{(r)}\left(\mathbb{C}_{0}\right)=-\infty$ when $\mathbb{C}_{0}$ is not positive definite. It then follows from the assumed concavity of $f^{(r)}$ that

$$
f^{(r)}(\mathbf{e})=\inf _{\mathbb{C}_{0}>0}\left\{\mathbb{C}_{0} \cdot \mathrm{e}-f_{*}^{(r)}\left(\mathbb{C}_{0}\right)\right\},
$$

where the restriction to positive definite $\mathbb{C}_{0}$ has been made since, otherwise, the righthand side would be infinite.

Using (2.7) in relation (2.12), it is concluded that

$$
w^{(r)}(\boldsymbol{\varepsilon})=\inf _{\mathbb{C}_{0}>0}\left\{\frac{1}{2} \boldsymbol{\varepsilon} \cdot \mathbb{C}_{0} \boldsymbol{\varepsilon}-f_{*}^{(r)}\left(\mathbb{C}_{0}\right)\right\} .
$$

It should be emphasized that this result, first given by Ponte Castañeda \& Suquet (1998), is valid for any 'concave extension' $f^{(r)}$ of the phase potential $w^{(r)}$, as provided by hypothesis 2.1 . 
Now, it follows from (2.11) that

$$
f_{*}^{(r)}\left(\mathbb{C}_{0}\right) \leq \inf _{\mathrm{e}=(1 / 2) \varepsilon \otimes \varepsilon}\left\{\mathbb{C}_{0} \cdot \mathrm{e}-f^{(r)}(\mathrm{e})\right\}=\inf _{\varepsilon}\left\{\frac{1}{2} \boldsymbol{\varepsilon} \cdot \mathbb{C}_{0} \boldsymbol{\varepsilon}-w^{(r)}(\boldsymbol{\varepsilon})\right\},
$$

and hence that

$$
-f_{*}^{(r)}\left(\mathbb{C}_{0}\right) \geq v^{(r)}\left(\mathbb{C}_{0}\right)
$$

where $v^{(r)}$ is the phase error function, as defined by (2.2). (It is important to emphasize that, in general, the functions $v^{(r)}$ cannot be identified with the functions $-f_{*}^{(r)}$.) Therefore, from expression (2.13), it is concluded that

$$
w^{(r)}(\boldsymbol{\varepsilon}) \geq \inf _{\mathbb{C}_{0}>0}\left\{\frac{1}{2} \boldsymbol{\varepsilon} \cdot \mathbb{C}_{0} \boldsymbol{\varepsilon}+v^{(r)}\left(\mathbb{C}_{0}\right)\right\} .
$$

But this expression, together with (2.6), which is itself independent of any concavity hypothesis, indeed implies that the identity (2.8) holds under the square concavity hypothesis 2.1 .

Next, recalling that $w$ is defined in terms of the phase potentials $w^{(r)}$ by relation (1.1), and defining $v$ similarly in terms of the functions $v^{(r)}$ by

$$
v\left(\boldsymbol{x}, \mathbb{C}_{0}\right)=\sum_{r=1}^{N} \chi^{(r)}(\boldsymbol{x}) v^{(r)}\left(\mathbb{C}_{0}\right),
$$

it is concluded that relations (2.9) and (2.10) also hold true, where use has been made of relation (2.1).

Remark 2.2. On account of the convexity of $v^{(r)}$ on $\mathbb{C}_{0}$, and of the fact that the restriction to positive definite $\mathbb{C}_{0}$ is artificial, the optimality condition in expression (2.8) for $w^{(r)}$ is given by the condition that zero should be included in the subdifferential of the terms in curly brackets, which may also be written as

$$
-\frac{1}{2} \boldsymbol{\varepsilon} \otimes \boldsymbol{\varepsilon} \in \mathrm{\partial}_{\mathbb{C}_{0}} v^{(r)}\left(\hat{\mathbb{C}}_{0}\right),
$$

where $\hat{\mathbb{C}}_{0}$ is the optimal value of $\mathbb{C}_{0}$, and $\partial_{\mathbb{C}_{0}} v^{(r)}$ is the subdifferential of the function $v^{(r)}$. In this connection, it should be emphasized that the functions $v^{(r)}$ are not smooth, even when the functions $w^{(r)}$ are. (This is essentially because the optimal solutions (2.3) in expressions (2.2) for the functions $v^{(r)}$ can change abruptly with changes in $\mathbb{C}_{0}$, leading to 'corners' and 'edges' in $v^{(r)}$.) As a consequence, the eigendirections of $\hat{\mathbb{C}}_{0}$ may be determined by these sharp edges, leaving the corresponding eigenvalues to be determined by appropriate stationarity conditions. In this connection, it is useful to note that the subdifferential of $v^{(r)}$, which is, by definition, a 'max-function', may be expressed as (e.g. Exercise 10 in section 6.1 of Borwein \& Lewis 2000)

$$
\partial_{\mathbb{C}_{0}} v^{(r)}\left(\mathbb{C}_{0}\right)=\left\{\mathrm{e} \mid \mathrm{e}=-\frac{1}{2} \sum_{m=1}^{M^{(r)}} a_{m}^{(r)}\left(\hat{\boldsymbol{\varepsilon}}_{(m)}^{(r)} \otimes \hat{\boldsymbol{\varepsilon}}_{(m)}^{(r)}\right), \quad a_{m}^{(r)} \geq 0, \quad \sum_{m=1}^{M^{(r)}} a_{m}^{(r)}=1\right\},
$$

where $\hat{\boldsymbol{\varepsilon}}_{(m)}^{(r)}, m=1, \ldots, M^{(r)}$, denote all the strain vectors for which the maximum in $(2.2)$ is attained for a given $\mathbb{C}_{0}$ (see also the optimality relations $(2.3)$ ). In other words, the subdifferential (2.19) of the function $v^{(r)}$ is the set of all convex combinations of the rank-one tensors $(-1 / 2) \hat{\boldsymbol{\varepsilon}}_{(m)}^{(r)} \otimes \hat{\boldsymbol{\varepsilon}}_{(m)}^{(r)}$, formed by the optimal strain vectors $\hat{\boldsymbol{\varepsilon}}_{(m)}^{(r)}$ in expression (2.2) for the functions $v^{(r)}$. 
Result 2.1. The stress-strain relation associated with the strain potential $w^{(r)}$, as determined by expression (2.8), is given by

$$
\boldsymbol{\sigma}=\frac{\partial w^{(r)}}{\partial \boldsymbol{\varepsilon}}(\boldsymbol{\varepsilon})=\hat{\mathbb{C}}_{0}(\boldsymbol{\varepsilon}) \boldsymbol{\varepsilon},
$$

where $\hat{\mathbb{C}}_{0}(\boldsymbol{\varepsilon})$ is the optimal value of $\mathbb{C}_{0}$ in expression $(2.8)$, evaluated at $\boldsymbol{\varepsilon}$.

Proof. Given the convexity of $w^{(r)}$ and the fact that the optimality condition in expression (2.8) is not a simple stationarity condition, it is best to work here with the subdifferential. Now, if $\boldsymbol{\sigma} \in \partial w^{(r)}(\boldsymbol{\varepsilon})$, then

$$
\boldsymbol{\sigma} \cdot\left(\boldsymbol{\varepsilon}^{\prime}-\boldsymbol{\varepsilon}\right) \leq w^{(r)}\left(\boldsymbol{\varepsilon}^{\prime}\right)-w^{(r)}(\boldsymbol{\varepsilon}), \text { for all } \boldsymbol{\varepsilon}^{\prime},
$$

or, using expression (2.8) for $w^{(r)}$,

$$
\begin{aligned}
\boldsymbol{\sigma} \cdot\left(\boldsymbol{\varepsilon}^{\prime}-\boldsymbol{\varepsilon}\right) & \leq \inf _{\mathbb{C}_{0}>0}\left\{\frac{1}{2} \boldsymbol{\varepsilon}^{\prime} \cdot \mathbb{C}_{0} \boldsymbol{\varepsilon}^{\prime}+v^{(r)}\left(\mathbb{C}_{0}\right)\right\}-\inf _{\mathbb{C}_{0}>0}\left\{\frac{1}{2} \boldsymbol{\varepsilon} \cdot \mathbb{C}_{0} \boldsymbol{\varepsilon}+v^{(r)}\left(\mathbb{C}_{0}\right)\right\} \\
& \leq \frac{1}{2} \boldsymbol{\varepsilon}^{\prime} \cdot \mathbb{C}_{0}^{\prime} \boldsymbol{\varepsilon}^{\prime}-v^{(r)}\left(\mathbb{C}_{0}^{\prime}\right)-\frac{1}{2} \boldsymbol{\varepsilon} \cdot \hat{\mathbb{C}}_{0} \boldsymbol{\varepsilon}+v^{(r)}\left(\hat{\mathbb{C}}_{0}\right), \text { for all } \boldsymbol{\varepsilon}^{\prime}, \mathbb{C}_{0}^{\prime}
\end{aligned}
$$

where $\hat{\mathbb{C}}_{0}$ is the optimal value of $\mathbb{C}_{0}$ evaluated at $\boldsymbol{\varepsilon}$. In particular, for $\mathbb{C}_{0}^{\prime}=\hat{\mathbb{C}}_{0}(\boldsymbol{\varepsilon})$, it follows that

$$
\boldsymbol{\sigma} \cdot\left(\boldsymbol{\varepsilon}^{\prime}-\boldsymbol{\varepsilon}\right) \leq \frac{1}{2} \boldsymbol{\varepsilon}^{\prime} \cdot \hat{\mathbb{C}}_{0}(\boldsymbol{\varepsilon}) \boldsymbol{\varepsilon}^{\prime}-\frac{1}{2} \boldsymbol{\varepsilon} \cdot \hat{\mathbb{C}}_{0}(\boldsymbol{\varepsilon}) \boldsymbol{\varepsilon}, \quad \text { for all } \boldsymbol{\varepsilon}^{\prime},
$$

which means that $\boldsymbol{\sigma} \in \partial w_{0}$, where $w_{0}=(1 / 2) \boldsymbol{\varepsilon} \cdot \hat{\mathbb{C}}_{0} \boldsymbol{\varepsilon}$, with $\hat{\mathbb{C}}_{0}$ fixed. But $w_{0}$ is differentiable, with derivative $\hat{\mathbb{C}}_{0} \boldsymbol{\varepsilon}$, and hence $\partial w_{0}(\boldsymbol{\varepsilon})=\left\{\hat{\mathbb{C}}_{0}(\boldsymbol{\varepsilon}) \boldsymbol{\varepsilon}\right\}$. In conclusion, it has been shown that if $\boldsymbol{\sigma} \in \partial w^{(r)}(\boldsymbol{\varepsilon})$, then $\boldsymbol{\sigma}=\hat{\mathbb{C}}_{0}(\boldsymbol{\varepsilon}) \boldsymbol{\varepsilon}$, which is the desired result.

Remark 2.3. Result 2.1 should not be confused with the optimality condition (2.3), which is only a prescription for the optimal $\hat{\boldsymbol{\varepsilon}}_{(m)}^{(r)}$, for some given $\mathbb{C}_{0}$, in the definition of the error function $v^{(r)}$. Interestingly, although expression (2.3) is commonly used to define (in a non-unique fashion) the secant modulus tensor (e.g. Ponte Castañeda \& Suquet 1998), it follows from result 2.1 that, in fact, a better definition of the secant modulus, in the general anisotropic case, is provided by the optimal $\hat{\mathbb{C}}_{0}$ in expression (2.8). This leads to a well-defined prescription that can be shown to be consistent with the standard prescription in the isotropic case.

It is also possible to obtain an alternative representation starting from the local stress potentials (2.2). To accomplish this, note that (1.4) can be re-expressed as

$$
\begin{aligned}
v^{(r)}\left(\mathbb{C}_{0}\right) & =\sup _{\boldsymbol{\varepsilon}}\left\{\sup _{\boldsymbol{\sigma}}\left\{\boldsymbol{\varepsilon} \cdot \boldsymbol{\sigma}-u^{(r)}(\boldsymbol{\sigma})\right\}-\frac{1}{2} \boldsymbol{\varepsilon} \cdot \mathbb{C}_{0} \boldsymbol{\varepsilon}\right\} \\
& =\sup _{\boldsymbol{\sigma}}\left\{\sup _{\boldsymbol{\varepsilon}}\left\{\boldsymbol{\varepsilon} \cdot \boldsymbol{\sigma}-\frac{1}{2} \boldsymbol{\varepsilon} \cdot \mathbb{C}_{0} \boldsymbol{\varepsilon}\right\}-u^{(r)}(\boldsymbol{\sigma})\right\} \\
& =\sup _{\boldsymbol{\sigma}}\left\{\frac{1}{2} \boldsymbol{\sigma} \cdot\left(\mathbb{C}_{0}\right)^{-1} \boldsymbol{\sigma}-u^{(r)}(\boldsymbol{\sigma})\right\},
\end{aligned}
$$


where use has been made of the fact that $w^{(r)}=u^{(r)^{*}}$ and of the positive definiteness of $\mathbb{C}_{0}$, as well as of the fact that the order of suprema can be interchanged. This allows the definition of a new function $\check{v}^{(r)}\left(\mathbb{S}_{0}\right)=v^{(r)}\left(\mathbb{C}_{0}=\mathbb{S}_{0}^{-1}\right)$, as follows.

Definition 2.2. The phase 'error' functions can alternatively be expressed in terms of the phase compliance tensors $\mathbb{S}_{0}$ via the relations

$$
\check{v}^{(r)}\left(\mathbb{S}_{0}\right)=\sup _{\boldsymbol{\sigma}}\left\{\frac{1}{2} \boldsymbol{\sigma} \cdot \mathbb{S}_{0} \boldsymbol{\sigma}-u^{(r)}(\boldsymbol{\sigma})\right\} .
$$

Remark 2.4. $\check{v}^{(r)}$ is a convex, non-negative function of $\mathbb{S}_{0}\left(=\mathbb{C}_{0}^{-1}\right)$, such that $\check{v}^{(r)}=0$ when $\mathbb{S}_{0} \leq 0$. Note that in the following there will be no risk of confusion, and, for simplicity, no attempt will be made to distinguish between $\check{v}^{(r)}$ and $v^{(r)}$, henceforth writing simply as $v^{(r)}\left(\mathbb{S}_{0}\right)$. Also, assuming smoothness of the functions $u^{(r)}$, the solutions of the non-concave optimization problem defined by (2.24) normally satisfy the stationarity conditions

$$
\mathbb{S}_{0} \hat{\boldsymbol{\sigma}}_{(m)}^{(r)}=\frac{\partial u^{(r)}}{\partial \boldsymbol{\sigma}}\left(\hat{\boldsymbol{\sigma}}_{(m)}^{(r)}\right)
$$

where $\hat{\boldsymbol{\sigma}}_{(m)}^{(r)}, m=1, \ldots, N^{(r)}$, denote all the stress tensors for which the maximum is attained in $(2.24)$ for a given $\mathbb{S}_{0}$.

Then, introducing the stress potential of the linear comparison material via

$$
u_{0}(\boldsymbol{x}, \boldsymbol{\sigma})=\frac{1}{2} \boldsymbol{\sigma} \cdot \mathbb{S}_{0}(\boldsymbol{x}) \boldsymbol{\sigma},
$$

it is also possible to define the local 'error' function

$$
v\left(\boldsymbol{x}, \mathbb{S}_{0}\right)=\sup _{\boldsymbol{\sigma}}\left\{u_{0}(\boldsymbol{x}, \boldsymbol{\sigma})-u(\boldsymbol{x}, \boldsymbol{\sigma})\right\} .
$$

From these definitions, the next result follows.

Lemma 2.2. Consider a composite with the phase stress potential $u^{(r)}=w^{(r)^{*}}$, as defined by relation (1.4), where $w^{(r)}$ are square concave (see hypothesis 2.1). Then, $u^{(r)}$ are 'square convex' and

$$
u^{(r)}(\boldsymbol{\sigma})=\sup _{\mathbb{S}_{0} \geq 0}\left\{\frac{1}{2} \boldsymbol{\sigma} \cdot \mathbb{S}_{0} \boldsymbol{\sigma}-v^{(r)}\left(\mathbb{S}_{0}\right)\right\}
$$

where the functions $v^{(r)}=\check{v}^{(r)}$ are defined by (2.24). Similarly, the local stress potential $u=w^{*}$, as defined by relation $(1.5)_{2}$, is given by

$$
u(\boldsymbol{x}, \boldsymbol{\sigma})=\sup _{\mathbb{S}_{0} \geq 0}\left\{u_{0}(\boldsymbol{x}, \boldsymbol{\sigma})-v\left(\boldsymbol{x}, \mathbb{S}_{0}\right)\right\}
$$

where $u_{0}$ and $v$ are the functions defined by (2.26) and (2.27), respectively.

Proof. On account of (2.8), it follows that

$$
\begin{aligned}
u^{(r)}(\boldsymbol{\sigma}) & =\sup _{\varepsilon}\left\{\boldsymbol{\varepsilon} \cdot \boldsymbol{\sigma}-\inf _{\mathbb{C}_{0}>0}\left\{\frac{1}{2} \boldsymbol{\varepsilon} \cdot \mathbb{C}_{0} \boldsymbol{\varepsilon}+v^{(r)}\left(\mathbb{C}_{0}\right)\right\}\right\} \\
& =\sup _{\mathbb{C}_{0}>0}\left\{\sup _{\boldsymbol{\varepsilon}}\left\{\boldsymbol{\varepsilon} \cdot \boldsymbol{\sigma}-\frac{1}{2} \boldsymbol{\varepsilon} \cdot \mathbb{C}_{0} \boldsymbol{\varepsilon}\right\}-v^{(r)}\left(\mathbb{C}_{0}\right)\right\} \\
& =\sup _{\mathbb{C}_{0}>0}\left\{\frac{1}{2} \boldsymbol{\sigma} \cdot\left(\mathbb{C}_{0}\right)^{-1} \boldsymbol{\sigma}-v^{(r)}\left(\mathbb{C}_{0}\right)\right\},
\end{aligned}
$$


which leads to result $(2.28)$ upon setting $\mathbb{S}_{0}=\mathbb{C}_{0}^{-1}$ (recall that $\mathbb{C}_{0}>0$ ). This result also demonstrates the existence of a convex function $g^{(r)}$ (in the space of fully symmetric, fourth-order tensors), such that

$$
u^{(r)}(\boldsymbol{\sigma})=g^{(r)}\left(\frac{1}{2} \boldsymbol{\sigma} \otimes \boldsymbol{\sigma}\right) .
$$

Thus, square concavity for $w^{(r)}$ implies square convexity for $u^{(r)}$. Note also that square convexity is consistent with 'super-quadratic' growth for $u^{(r)}$. The derivation of $(2.29)$ is now straightforward in view of $(1.5)_{2},(2.17)$ and $(2.26)$.

\section{Variational principles for anisotropic materials}

In this section, use is made of expressions (2.8) and (2.28) for the local potentials $w$ and $u$ in terms of linear comparison materials to derive alternative variational representations for $\tilde{w}$ and $\tilde{u}$ that are equivalent to $(1.2)$ and $(1.6)_{2}$. These representations have the advantage that they can be used to generate improved bounds - relative to the classical bounds of Voigt and Reuss - for the effective potentials.

Proposition 3.1. Under the 'square concavity' hypothesis 2.1, the effective potential $\tilde{w}$ defined by (1.2) admits the alternative representation

$$
\tilde{w}(\overline{\boldsymbol{\varepsilon}})=\inf _{\mathbb{C}_{0}(\boldsymbol{x})>0}\left\{\tilde{w}_{0}(\overline{\boldsymbol{\varepsilon}})+\mathcal{V}\left(\mathbb{C}_{0}\right)\right\},
$$

where $\mathcal{V}\left(\mathbb{C}_{0}\right)=\left\langle v\left(\boldsymbol{x}, \mathbb{C}_{0}(\boldsymbol{x})\right\rangle\right.$, with $v$ given by (2.10) and $\tilde{w}_{0}$ denotes the effective potential of the linear comparison material with local potential (2.1), i.e.

$$
\tilde{w}_{0}(\overline{\boldsymbol{\varepsilon}})=\inf _{\varepsilon \in \mathcal{K}(\overline{\boldsymbol{\varepsilon}})}\left\langle w_{0}(\boldsymbol{x}, \boldsymbol{\varepsilon})\right\rangle .
$$

Proof. Lemma 2.1 is used together with expression (1.2) to deduce the following result for the effective strain-energy density:

$$
\tilde{w}(\overline{\boldsymbol{\varepsilon}})=\inf _{\boldsymbol{\varepsilon} \in \mathcal{K}(\overline{\boldsymbol{\varepsilon}})} \inf _{\mathbb{C}_{0}(\boldsymbol{x})>0}\left\{\left\langle w_{0}(\boldsymbol{x}, \boldsymbol{\varepsilon})\right\rangle+\left\langle v\left(\boldsymbol{x}, \mathbb{C}_{0}(\boldsymbol{x})\right\rangle\right\},\right.
$$

where the infimum over $\mathbb{C}_{0}$ has been taken out of the volume integral implied by the triangular brackets. (Note that $\mathbb{C}_{0}$ is now a function of position $\boldsymbol{x}$.) But the order of infima can be interchanged, and hence

$$
\tilde{w}(\overline{\boldsymbol{\varepsilon}})=\inf _{\mathbb{C}_{0}(\boldsymbol{x})>0} \inf _{\varepsilon \in \mathcal{K}(\overline{\boldsymbol{\varepsilon}})}\left\{\left\langle w_{0}(\boldsymbol{x}, \boldsymbol{\varepsilon})\right\rangle+\left\langle v\left(\boldsymbol{x}, \mathbb{C}_{0}(\boldsymbol{x})\right\rangle\right\} .\right.
$$

Noticing that the inner infimum over $\varepsilon$ affects only the first term inside the curly brackets, and that $\mathcal{V}\left(\mathbb{C}_{0}\right)=\left\langle v\left(\boldsymbol{x}, \mathbb{C}_{0}(\boldsymbol{x})\right\rangle\right.$, one arrives at the desired result.

Remark 3.1. The variational representation (3.1) generalizes a corresponding variational representation for nonlinear composites with isotropic phases first given by Ponte Castañeda (1992). It expresses the effective properties of the nonlinear composite (through its potential $\tilde{w}$ ) in terms of two functionals. The functional $\tilde{w}_{0}$ is the elastic energy of a fictitious linear heterogeneous solid, called the linear comparison material, made up of phases with stiffness $\mathbb{C}_{0}(\boldsymbol{x})$ at a point $\boldsymbol{x}$, whose properties are determined by the solution of the variational representation (3.1) itself. The functional $\mathcal{V}$ depends on the phase error functions 
$v^{(r)}$, and thus provides a measure of the nonlinearity of the actual material. The representation (3.1) is exact and strictly equivalent to the variational characterization of $\tilde{w}$ given in (1.2). But it requires the exact solution of the homogenization problem (3.2), which is a difficult task in view of the fact that it corresponds to a linear composite material with infinitely many phases. However, as will be seen in $\S 4$, this variational representation can be used to generate estimates for $\tilde{w}$, by making use of suitable trial fields for the moduli tensor $\mathbb{C}_{0}$ of the linear comparison material.

Remark 3.2. Representation (3.1) also provides an interpretation of the strain field in the actual nonlinear composite as the strain field in the optimal linear comparison solid. Indeed, although the strain field $\varepsilon_{0}$ associated with the minimizer in the linear comparison problem (3.2) depends on $\mathbb{C}_{0}$, and is therefore different, in general, from the strain field $\varepsilon$ associated with the original nonlinear problem (1.2), under the hypothesis of square concavity of $w$, it follows from (3.1) that the 'linear' minimizer $\varepsilon_{0}$ arising from the optimal choice of $\mathbb{C}_{0}$ is precisely the nonlinear minimizer $\varepsilon$.

It is also possible to start from the identity (2.29) for the local stress potential $u$ to generate a corresponding representation for the effective stress potential $\tilde{u}$.

Proposition 3.2. Assuming 'square convexity' of the local stress potential u (or 'square concavity' of the corresponding strain potential $w$ ), the effective stress potential $\tilde{u}$ defined by $(1.6)_{2}$ admits the alternative representation

$$
\tilde{u}(\overline{\boldsymbol{\sigma}})=\sup _{\mathbb{S}_{0}(\boldsymbol{x}) \geq 0}\left\{\tilde{u}_{0}(\overline{\boldsymbol{\sigma}})-\mathcal{V}\left(\mathbb{S}_{0}\right)\right\},
$$

where $\mathcal{V}\left(\mathbb{S}_{0}\right)=\left\langle v\left(\boldsymbol{x}, \mathbb{S}_{0}(\boldsymbol{x})\right\rangle\right.$, with $v$ given by (2.27) and $\tilde{u}_{0}$ denotes the effective potential of the linear comparison material with local potential (2.26), i.e.

$$
\tilde{u}_{0}(\overline{\boldsymbol{\sigma}})=\inf _{\boldsymbol{\sigma} \in \mathcal{S}(\overline{\boldsymbol{\sigma}})}\left\langle u_{0}(\boldsymbol{x}, \boldsymbol{\sigma})\right\rangle .
$$

Proof. Lemma 2.2 is used together with expression $(1.6)_{2}$ to deduce the following result for the effective stress potential:

$$
\tilde{u}(\overline{\boldsymbol{\sigma}})=\inf _{\boldsymbol{\sigma} \in \mathcal{S}(\overline{\boldsymbol{\sigma}})} \sup _{\mathbb{S}_{0}(\boldsymbol{x}) \geq 0}\left\{\left\langle u_{0}(\boldsymbol{x}, \boldsymbol{\sigma})\right\rangle-\left\langle v\left(\boldsymbol{x}, \mathbb{S}_{0}(\boldsymbol{x})\right\rangle\right\},\right.
$$

where the supremum over $\mathbb{S}_{0}$ has been taken out of the triangular brackets. But $u_{0}$ is convex in $\boldsymbol{\sigma}$ and $-v$ is concave in $\mathbb{S}_{0}$, and, therefore, by the saddle point theorem (Ekeland \& Teman 1999), the order of the infimum and supremum can be interchanged, and hence

$$
\tilde{u}(\overline{\boldsymbol{\sigma}})=\sup _{\mathbb{S}_{0}(\boldsymbol{x}) \geq 0} \inf _{\boldsymbol{\sigma} \in \mathcal{S}(\overline{\boldsymbol{\sigma}})}\left\{\left\langle u_{0}(\boldsymbol{x}, \boldsymbol{\sigma})\right\rangle-\left\langle v\left(\boldsymbol{x}, \mathbb{S}_{0}(\boldsymbol{x})\right\rangle\right\},\right.
$$

which leads to the desired result.

Remark 3.3. It can be shown directly that the two versions of the variational representations, (3.1) and (3.5), are exactly equivalent, under the given hypothesis on the potentials $w$ and $u$, respectively. (In other words, result (3.5) for $\tilde{u}$ can be shown to follow directly from result (3.1) for $\tilde{w}$ by using the fact that $\tilde{u}=\tilde{w}^{*}$.) It should also be emphasized that the variational principles (3.1) and (3.5) for composites with anisotropic phases are entirely consistent with the 
earlier variational representations (Ponte Castañeda 1992) for composites with isotropic phases. Indeed, it can be shown that, for isotropic nonlinear composites, the optimal choice for the comparison elasticity and the compliance tensors in the context of the general anisotropic forms (3.1) and (3.5) is provided by isotropic fourth-order tensors.

Remark 3.4. Finally, it is noted that an alternative version of the variational representations (3.1) and (3.5) for composites with anisotropic phases was presented by Ponte Castañeda \& Suquet (1998), generalizing earlier estimates for the special case of crystalline plasticity by deBotton \& Ponte Castañeda (1995). These variational principles have a form identical to the representations (3.1) and (3.5), except that the functions $v^{(r)}$ are defined differently. Essentially, $v^{(r)}$ are identified with the functions $-f_{*}^{(r)}$ introduced in expressions $(2.11)$, which, as has already been observed (see equations (2.15)), are different, in general, from the functions $v^{(r)}$, as defined by relations $(2.2)$.

\section{Bounds and estimates via piecewise constant moduli}

Unlike the stress and strain trial fields in the context of the classical variational representations (1.2) and $(1.6)_{2}$, the trial fields of stiffnesses and compliances in the variational representations (3.1) and (3.5) can be chosen to be constant in each phase (not necessarily the same constant). Thus, the optimization over the elasticity moduli $\mathbb{C}_{0}(\boldsymbol{x})$ can be restricted to the set of piecewise constant moduli

$$
\mathbb{C}_{0}(\boldsymbol{x})=\sum_{r=1}^{N} \chi^{(r)}(\boldsymbol{x}) \mathbb{C}_{0}^{(r)}
$$

where the tensors $\mathbb{C}_{0}^{(r)}$ are taken to be constant. Making use of this trial field in the variational statement (3.1) for $\tilde{w}$ then leads to the following bound, which is a generalization for composites with anisotropic phases of bounds that were first given for composites with isotropic phases by Ponte Castañeda (1991).

Result 4.1. The effective strain potential $\tilde{w}$ of the nonlinear composite is bounded above by

$$
\tilde{w}_{+}(\overline{\boldsymbol{\varepsilon}})=\inf _{\substack{\mathbb{C}_{0}^{(r)}>0 \\ r=1, \ldots, N}}\left\{\tilde{w}_{0}(\overline{\boldsymbol{\varepsilon}})+\sum_{r=1}^{N} c^{(r)} v^{(r)}\left(\mathbb{C}_{0}^{(r)}\right)\right\},
$$

where the functions $v^{(r)}$ are defined by relations $(2.2)$, and

$$
\tilde{w}_{0}(\overline{\boldsymbol{\varepsilon}})=\frac{1}{2} \overline{\boldsymbol{\varepsilon}} \cdot \tilde{\mathbb{C}}_{0}\left(\mathbb{C}_{0}^{(s)}\right) \overline{\boldsymbol{\varepsilon}}
$$

is now the effective strain potential (3.2) of a linear comparison composite (LCC) with the uniform stiffness tensors $\mathbb{C}_{0}^{(r)}$ in each of the phases $(r=1, \ldots, N)$-and, hence, the same microstructure as the nonlinear composite - and with the effective stiffness tensor $\tilde{\mathbb{C}}_{0}$.

Remark 4.1. The form (4.2) for the bound $\tilde{w}_{+}(\overline{\boldsymbol{\varepsilon}})$ involves a convex optimization problem for the stiffness tensors $\mathbb{C}_{0}^{(r)}$. This follows, for example, from lemma 2.1 of Ekeland \& Temam (1999), because the terms inside the curly 
brackets may be written as the infimum (over the variables $\varepsilon \in \mathcal{K}(\overline{\boldsymbol{\varepsilon}})$ ) of a convex function in the arguments $\varepsilon$ and $\mathbb{C}_{0}^{(r)}$ (refer to equation (3.3)). In this connection, it is important to recall that the tensors $\mathbb{C}_{0}^{(r)}$ need not be restricted to be positive definite (cf. the passage from relations (2.5) to (2.6)); it is simply that the infimum cannot occur for $\mathbb{C}_{0}^{(r)}$ that are not positive definite, since the value of the functions $v^{(r)}$ would be infinite in that case. Thus, given that the optimization problem for the bound $\tilde{w}_{+}$is convex for the whole space of stiffness tensors $\mathbb{C}_{0}^{(r)}$ - and the fact that the functions $v^{(r)}$ need not be smooth - a necessary and sufficient condition for the minimum to be attained is that zero be included in the subdifferential of the terms inside the curly brackets in equation (4.2), for the optimal stiffness tensors $\hat{\mathbb{C}}_{0}^{(r)}$. Now, since $\tilde{w}_{0}$ is differentiable (with respect to $\left.\mathbb{C}_{0}^{(r)}\right)$, this optimality condition can also be written as

$$
-\frac{1}{2 c^{(r)}} \frac{\partial\left(\overline{\boldsymbol{\varepsilon}} \cdot \tilde{\mathbb{C}}_{0} \overline{\boldsymbol{\varepsilon}}\right)}{\partial \mathbb{C}_{0}^{(r)}} \in \partial_{\mathbb{C}_{0}} v^{(r)}\left(\hat{\mathbb{C}}_{0}^{(r)}\right) .
$$

Then, making use of the following identity for linear composites (e.g. Parton \& Buryachenko 1990)

$$
\left\langle\varepsilon_{\mathrm{L}} \otimes \varepsilon_{\mathrm{L}}\right\rangle^{(r)}=\frac{1}{c^{(r)}} \frac{\partial\left(\overline{\boldsymbol{\varepsilon}} \cdot \tilde{\mathbb{C}}_{0} \overline{\boldsymbol{\varepsilon}}\right)}{\partial \mathbb{C}_{0}^{(r)}},
$$

where $\boldsymbol{\varepsilon}_{\mathrm{L}}$ is the local strain field in the LCC, as well as of expression (2.19) for the subdifferential of $v^{(r)}$, the optimality conditions (4.4) for $\mathbb{C}_{0}^{(r)}$ may also be expressed as follows: do there exist an integer $M^{(r)}$ and constants $a_{m}^{(r)}\left(m=1, \ldots, M^{(r)}\right)$, satisfying the conditions $a_{m}^{(r)} \geq 0, \sum_{m=1}^{M^{(r)}} a_{m}^{(r)}=1$, such that the identity

$$
\left\langle\boldsymbol{\varepsilon}_{\mathrm{L}} \otimes \boldsymbol{\varepsilon}_{\mathrm{L}}\right\rangle^{(r)}=\sum_{m=1}^{M^{(r)}} a_{m}^{(r)}\left(\hat{\boldsymbol{\varepsilon}}_{(m)}^{(r)} \otimes \hat{\boldsymbol{\varepsilon}}_{(m)}^{(r)}\right),
$$

is satisfied? Thus, it is seen here too that the optimal values $\hat{\mathbb{C}}_{0}^{(r)}$ may be related to the second moments $\left\langle\varepsilon_{\mathrm{L}} \otimes \varepsilon_{\mathrm{L}}\right\rangle^{(r)}$ of the strain field in the LCC, as has been shown by Suquet (1995) for the special case of composites with isotropic phases. In general, the tensor of the second moments of the strain is of full rank, and, consequently, the optimality conditions (4.6) require that $\hat{\mathbb{C}}_{0}^{(r)}$ be such that the maximum in the function $v^{(r)}$ be attained simultaneously at several strains $\hat{\boldsymbol{\varepsilon}}_{(m)}^{(r)}$ (i.e. $M^{(r)}$ should be sufficiently large), so that the fourth-order tensors on the two sides of (4.6) have the same rank. In turn, this implies that the optimal $\hat{\mathbb{C}}_{0}^{(r)}$ should be precisely in regions where the functions $v^{(r)}$ are not differentiable $\left(M^{(r)}>1\right)$. Thus, the fact that the functions $v^{(r)}$ have sharp edges implies that the optimal tensors $\hat{\mathbb{C}}_{0}^{(r)}$ have certain preferred orientations, depending on the anisotropy of the functions $w^{(r)}$, and correspondingly depend only on certain traces of the second moment strain tensor $\left\langle\varepsilon_{\mathrm{L}} \otimes \varepsilon_{\mathrm{L}}\right\rangle^{(r)}$.

Remark 4.2. It is also possible to make use of expressions (2.2) for the functions $v^{(r)}$ in expression (4.2) for $\tilde{w}_{+}$, to rewrite the problem as an inf-sup optimization. (Note that this problem is not concave in the variables $\boldsymbol{\varepsilon}$, and therefore the order of the inf and the sup cannot be interchanged in general.) Then, using the optimality conditions (4.6), it can be shown that the bound $\tilde{w}_{+}$ 
can be formally written in the form

$$
\tilde{w}_{+}(\overline{\boldsymbol{\varepsilon}})=\sum_{r=1}^{N} \sum_{m=1}^{M^{(r)}} c^{(r)} a_{m}^{(r)} w^{(r)}\left(\hat{\boldsymbol{\varepsilon}}_{(m)}^{(r)}\right),
$$

where $\hat{\boldsymbol{\varepsilon}}_{(m)}^{(r)}$ are the optimal solutions of problem $(2.2)$ for the functions $v_{(r)}^{(r)}$, satisfying the secant condition (2.3), and evaluated at the optimal values of $\hat{\mathbb{C}}_{0}^{(r)}$, as determined by relation (4.2). This form generalizes the form proposed by Suquet (1995) for composites with isotropic phases, but it should be emphasized that in the more general case of anisotropic phases the (difficult) inf-sup problem must be solved anyway to determine the correct choice for $\hat{\boldsymbol{\varepsilon}}_{(m)}^{(r)}$.

In general, the estimate (4.2) for $\tilde{w}$ requires numerical treatment, and therefore to determine the associated stress directly from this expression, the relevant derivative with respect to $\overline{\boldsymbol{\varepsilon}}$ would have to be computed numerically. However, the following result avoids this complication.

Result 4.3. The effective stress-strain relation of the nonlinear composite is approximated by

$$
\overline{\boldsymbol{\sigma}}=\frac{\partial \tilde{w}_{+}}{\partial \overline{\boldsymbol{\varepsilon}}}(\overline{\boldsymbol{\varepsilon}})=\tilde{\mathbb{C}}_{0}\left(\hat{\mathbb{C}}_{0}^{(s)}\right) \overline{\boldsymbol{\varepsilon}},
$$

where $\hat{\mathbb{C}}_{0}^{(s)}$ are the stiffness tensors satisfying the optimality conditions in (4.2).

Remark 4.4. The demonstration of this result is analogous to the derivation of result 2.1, and will not be detailed further here for conciseness. It should be emphasized, however, that relation (4.8) is an approximation to the exact stress-strain relation of the nonlinear composite. This relation is a generalization of a result first given in the context of composites with isotropic phases by deBotton \& Ponte Castañeda (1993). In spite of its appearance, this effective stress-strain relation is nonlinear, due to the nonlinear dependence of the optimal $\hat{\mathbb{C}}_{0}^{(r)}$ on the average strain $\overline{\boldsymbol{\varepsilon}}$. In fact, in parallel with remark 2.3 concerning the interpretation of $\hat{\mathbb{C}}_{0}^{(s)}$ as the secant moduli tensors of the phases, relation (4.8) suggests that $\tilde{\mathbb{C}}_{0}$ can be interpreted as the secant modulus tensor of the nonlinear composite, generalizing earlier interpretations for the isotropic case by Suquet (1995).

A bound that is equivalent to (4.2) can be obtained by considering the stress potential $\tilde{u}$ and its variational representation (3.5). Thus, restricting the optimization in (3.5) to piecewise constant compliances $\mathbb{S}_{0}^{(r)}$ in the variational representation (3.5) leads to a lower bound for $\tilde{u}$. In addition, an estimate for the stress-strain relation may be obtained by differentiation, which is completely analogous to the estimate (4.8) in result 4.3 .

Result 4.4. The effective stress potential $\tilde{u}$ of the nonlinear composite is bounded below by

$$
\tilde{u}_{-}(\overline{\boldsymbol{\sigma}})=\sup _{\substack{\mathbb{S}_{0}^{(r)} \geq 0 \\ r=1, \ldots, N}}\left\{\tilde{u}_{0}(\overline{\boldsymbol{\sigma}})-\sum_{r=1}^{N} c^{(r)} v^{(r)}\left(\mathbb{S}_{0}^{(r)}\right)\right\},
$$


where the functions $v^{(r)}$ have been defined by relations (2.24), and

$$
\tilde{u}_{0}(\overline{\boldsymbol{\sigma}})=\frac{1}{2} \overline{\boldsymbol{\sigma}} \cdot \tilde{\mathbb{S}}_{0}\left(\mathbb{S}_{0}^{(s)}\right) \overline{\boldsymbol{\sigma}}
$$

is now the effective stress potential of a linear comparison composite (LCC) with uniform compliance tensors $\mathbb{S}_{0}^{(r)}$ in each of the phases $(r=1, \ldots, N)$ and the effective compliance tensor $\tilde{\mathbb{S}}_{0}$. Moreover, the following estimate is obtained for the effective stress-strain relation of the nonlinear composite

$$
\overline{\boldsymbol{\varepsilon}}=\frac{\partial \tilde{u}_{-}}{\partial \overline{\boldsymbol{\sigma}}}(\overline{\boldsymbol{\sigma}})=\tilde{\mathbb{S}}_{0}\left(\hat{\mathfrak{S}}_{0}^{(s)}\right) \overline{\boldsymbol{\sigma}}
$$

where $\hat{\mathbb{S}}_{0}^{(s)}$ are the optimal values of $\mathbb{S}_{0}^{(s)}$ in expression (4.9).

Remark 4.5. Expression (4.9) for the bound $\tilde{u}_{-}$involves a concave optimization problem for the compliance tensors $\mathbb{S}_{0}^{(r)}$. This follows from the facts that the functions $v^{(r)}$ are convex in the tensors $\mathbb{S}_{0}^{(r)}$, while the term arising from $\tilde{u}_{0}$ is, on the other hand, concave in the tensors $\mathbb{S}_{0}^{(r)}$. (This last observation is easy to show making use of definition (3.6) and of the fact that the infimum of the sum is greater than the sum of the infima.) Then, the analysis carried out in the context of remarks 4.1 and 4.2 can be repeated step by step, with results completely analogous to expressions (4.4), (4.6) and (4.7).

\section{(a) Special results for power-law materials}

The above bounds for the effective potential may be given simpler, alternative forms for power-law composites by exploiting the homogeneity of the relevant potentials and error functions. Such results generalize earlier results for composites with isotropic phases by Suquet (1993), who made use of Hölder's inequality for their derivation. Thus, when phase $r$ is of the (incompressible) power-law type with exponent $m$, such that $0 \leq m \leq 1$, the strain potential $w^{(r)}$ is positively homogeneous of degree $m+1$,

$$
w^{(r)}(\lambda \boldsymbol{\varepsilon})=\lambda^{m+1} w^{(r)}(\boldsymbol{\varepsilon}), \quad \forall \lambda \geq 0 .
$$

Replacing $\varepsilon$ by $\lambda \boldsymbol{\varepsilon}$ in expression (2.2), using property (4.12), and optimizing with respect to $\lambda$, the function $v^{(r)}$ can be written in the form

$$
v^{(r)}\left(\mathbb{C}_{0}\right)=\frac{1-m}{2(1+m)} \sup _{\varepsilon}\left\{\frac{\left[(1+m) w^{(r)}(\boldsymbol{\varepsilon})\right]^{2 /(1-m)}}{\left[\varepsilon \cdot \mathbb{C}_{0} \boldsymbol{\varepsilon}\right]^{(1+m) /(1-m)}}\right\},
$$

where the optimization variables $\varepsilon$ can now be chosen to have magnitude 1 (and therefore belong to a bounded space). Note that it follows from this expression that $v^{(r)}$ is a homogeneous function of degree $(m+1) /(m-1)$ in the variable $\mathbb{C}_{0}$.

When all the phases in a composite are made of (incompressible) power-law materials with the same exponent $m$, the composite is itself an (incompressible) power-law material (Ponte Castañeda \& Suquet 1998). In other words, the effective potential $\tilde{w}$ is also homogeneous of degree $m+1$. Then, letting $\mathbb{C}_{0}(\boldsymbol{x})=t \mathbb{C}_{0}(\boldsymbol{x})$, for arbitrary positive $t$, noting that $\tilde{w}_{0}$ and $v^{(r)}$, as defined by relations (4.3) and (4.13), are homogeneous functions of degrees 1 and $(m+1)$ / $(m-1)$ in $\mathbb{C}_{0}$, respectively, and optimizing with respect to $t$, the following alternative representation for the bound $\tilde{w}_{+}$is obtained. 
Result 4.5. The effective strain potential $\tilde{w}$ for power-law composites is bounded above by

$$
\tilde{w}_{+}(\overline{\boldsymbol{\varepsilon}})=\frac{2}{m+1} \inf _{\substack{\mathbb{C}_{0}^{(r)}>0 \\ r=1, \ldots, N}}\left\{\left[\tilde{w}_{0}(\overline{\boldsymbol{\varepsilon}})\right]^{(m+1) / 2}\left[\frac{1+m}{1-m} \sum_{r=1}^{N} c^{(r)} v^{(r)}\left(\mathbb{C}_{0}^{(r)}\right)\right]^{(1-m) / 2}\right\},
$$

where $v^{(r)}$ are given by (4.13).

A corresponding representation can be given for the lower bound $\tilde{u}_{-}$when all the individual phases are power-law materials with the same exponent $n=1 / \mathrm{m}$. The details are omitted here for brevity, but the result is as follows.

Result 4.6. The effective stress potential $\tilde{u}$ for power-law composites is bounded below by

$$
\tilde{u}_{-}(\overline{\boldsymbol{\sigma}})=\frac{2}{n+1} \sup _{\substack{\mathbb{S}_{0}^{(r)}>0 \\ r=1, \ldots, N}}\left\{\left[\tilde{u}_{0}(\overline{\boldsymbol{\sigma}})\right]^{(n+1) / 2}\left[\frac{n+1}{n-1} \sum_{r=1}^{N} c^{(r)} v^{(r)}\left(\mathbb{S}_{0}^{(r)}\right)\right]^{(1-n) / 2}\right\}
$$

where

$$
v^{(r)}\left(\mathbb{S}_{0}\right)=\frac{n-1}{2(n+1)} \sup _{\boldsymbol{\sigma}}\left\{\frac{\left[\boldsymbol{\sigma} \cdot \mathbb{S}_{0} \boldsymbol{\sigma}\right]^{(n+1) /(n-1)}}{\left[(n+1) u^{(r)}(\boldsymbol{\sigma})\right]^{2 /(n-1)}}\right\} .
$$

\section{(b) Special results for rigid-ideally plastic materials}

Even though simpler than the general form of the bounds, the alternative forms of the bounds (4.14) and (4.15) for power-law composites still require the solution of difficult non-concave problems (4.13) and (4.16) for the functions $v^{(r)}$. In the limiting case of ideal plasticity $(n \rightarrow \infty)$, however, this problem simplifies considerably. This is because, in that case, the functions $v^{(r)}$, defined by $(2.24)$, can be written in the form

$$
v^{(r)}\left(\mathbb{S}_{0}\right)=\sup _{\boldsymbol{\sigma} \in P^{(r)}}\left\{\frac{1}{2} \boldsymbol{\sigma} \cdot \mathbb{S}_{0} \boldsymbol{\sigma}\right\},
$$

where $P^{(r)}$ is the strength domain of phase $r$, defined by the conditions $u^{(r)}(\boldsymbol{\sigma})=0$ if $\boldsymbol{\sigma} \in P^{(r)}$, and $\infty$ otherwise. Note that $P^{(r)}$ is the closed, convex set of stress tensors bounded by the yield surface of phase $r$. The advantage of rewriting the functions $v^{(r)}$ in this way is that its computation reduces to the well-studied problem of finding the maximum of a convex function relative to a convex set. Indeed, it can be shown (see corollary 32.3.2 in Rockafellar 1970) that the maximum in (4.17) is attained at one or more of the extreme points of the set $P^{(r)}$, which means that the optimal $\hat{\boldsymbol{\sigma}}_{(m)}^{(r)}$ are necessarily on the yield surface of phase $r$. Furthermore, in many cases, such as that of crystalline phases considered in part II of this work, the number of extreme points of $P^{(r)}$ is finite, 
and so the function $v^{(r)}$ can be written as

$$
v^{(r)}\left(\mathbb{S}_{0}\right)=\max _{k=1, \ldots, K_{e}^{(r)}}\left\{\frac{1}{2} \boldsymbol{\sigma}_{(k)}^{(r)} \cdot \mathbb{S}_{0} \boldsymbol{\sigma}_{(k)}^{(r)}\right\}
$$

where $K_{e}^{(r)}$ is the total number of extreme points of $P^{(r)}$, and $\boldsymbol{\sigma}_{(k)}^{(r)}$ denotes the stress vector associated with the $k$ th extreme point. For a given $\mathbb{S}_{0}$, this expression is very simple to evaluate. Finally, considering the limit as $n \rightarrow \infty$ in expression (4.15) for $\tilde{u}_{-}$, the following result is obtained for the effective strength domain $\tilde{P}$ of the ideally plastic composite, which is defined by the conditions $\tilde{u}(\overline{\boldsymbol{\sigma}})=0$ if $\overline{\boldsymbol{\sigma}} \in \tilde{P}$, and $\infty$ otherwise.

Result 4.7. The effective strength domain $\tilde{P}$ of the ideally plastic composite is bounded from the outside by

$$
\tilde{P}_{+}=\left\{\overline{\boldsymbol{\sigma}} \mid \tilde{u}_{0}(\overline{\boldsymbol{\sigma}}) \leq \sum_{r=1}^{N} c^{(r)} v^{(r)}\left(\mathbb{S}_{0}^{(r)}\right), \quad \forall \mathbb{S}_{0}^{(r)} \geq 0\right\} .
$$

Note that this set corresponds to the intersection of all quadratic functions of the average stress $\overline{\boldsymbol{\sigma}}$, which are defined by the conditions $\tilde{u}_{0}(\overline{\boldsymbol{\sigma}}) \leq \sum_{r=1}^{N} c^{(r)} v^{(r)}\left(\mathbb{S}_{0}^{(r)}\right)$, for each possible choice of the compliance tensors $\mathbb{S}_{0}^{(r)} \geq 0$ of the LCC.

\section{Concluding remarks}

In $\S 4$, bounds have been derived for nonlinear composites with anisotropic phases, including special forms for power-law and ideally plastic composites, making use of the variational representations derived in §3. As already noted (remark 3.4), alternative forms for the variational representations have been proposed by Ponte Castañeda \& Suquet (1998). These alternative representations for $\tilde{w}$ and $\tilde{u}$ can be used to generate bounds that exhibit precisely the same forms as bounds (4.2) and (4.9) for general square concave and square convex phase potentials $w^{(r)}$ and $u^{(r)}$, respectively, except that the functions $v^{(r)}$, as defined by relations (2.2) (or (2.24)), must be replaced by the functions $-f_{*}^{(r)}$, as defined in expression (2.11). Similarly, special forms of the bounds may be derived for power-law and ideally plastic composites that correspond exactly to the forms (4.14), (4.15) and (4.19), but with the same caveat for the functions $v^{(r)}$.

Now, it follows trivially from inequalities $(2.15), v^{(r)} \leq-f_{*}^{(r)}$, that the bounds proposed in this work are at least as good as the corresponding bounds given by Ponte Castañeda \& Suquet (1998). The question of interest, however, is whether the bounds given in this work are actually better, in general, as suggested by the above-mentioned inequality. This question will be partially answered in part II of this work in the context of composites with crystalline phases. In fact, for the specific class of crystalline materials, the bounds of Ponte Castañeda \& Suquet (1998) have already been shown to recover exactly (for some appropriate choice of the 'extension' functions $f^{(r)}$ ) the bounds of deBotton \& Ponte Castañeda (1995). In part II, it will be shown that the new bounds derived in this work are sharper, in general, than the bounds of 
deBotton \& Ponte Castañeda (1995) for crystalline materials. This suggests that the new bounds may also be sharper, in general, than the bounds of Ponte Castañeda \& Suquet (1998).

It should be emphasized, however, that the earlier bounds have a significant advantage over the new bounds proposed in this work in terms of computational efficiency. Indeed, the error functions $v^{(r)}$ needed in the computation of the new bounds require solving a non-concave (non-convex) problem, while the corresponding functions $-f_{*}^{(r)}$ in the earlier bounds require the solution of a concave problem (in an enlarged space). This additional structure, which makes the problem for the bounds concave in one set of variables and convex in the other, allows the use of the saddle point theorem to simplify the computation of the earlier bounds. In particular, the form of the bounds corresponding to expression (4.7) depends directly on the second moments of the strain in the linear comparison composite. (On the other hand, the earlier bounds could depend on the concave extension $f^{(r)}$ of the phase potentials $w^{(r)}$, while the new ones, given in this work, depend only on the actual potentials $w^{(r)}$.) Thus, in conclusion, the new bounds developed in this work can give improved results, relative to the earlier bounds, but any improvement will come at an increased computational cost.

This paper is based upon the work supported by the National Science Foundation under grants CMS-02-01454 and OISE-02-31867.

\section{References}

Borwein, J. M. \& Lewis, A. S. 2000 Convex analysis and nonlinear optimization. New York, NY: Springer.

deBotton, G. \& Ponte Castañeda, P. 1993 Elastoplastic constitutive relations for fiber-reinforced solids. Int. J. Solids Struct. 30, 1865-1890. (doi:10.1016/0020-7683(93)90222-S)

deBotton, G. \& Ponte Castañeda, P. 1995 Variational estimates for the creep behaviour of polycrystals. Proc. R. Soc. A 448, 121-142.

Ekeland, I. \& Temam, R. 1999 Convex analysis and variational problems. Philadelphia, PA: SIAM.

Garroni, A. \& Kohn, R. V. 2003 Some three-dimensional problems related to dielectric breakdown and polycrystal plasticity. Proc. R. Soc. A 459, 2613-2625. (doi:10.1098/rspa.2003.1152)

Goldsztein, G. H. 2001 Rigid perfectly plastic two-dimensional polycrystals. Proc. R. Soc. A 457, 2789-2798. (doi:10.1098/rspa.2001.0839)

Hashin, Z. \& Shtrikman, S. 1963 A variational approach to the theory of the elastic behavior of multiphase materials. J. Mech. Phys. Solids 11, 127-140. (doi:10.1016/0022-5096(63)90060-7)

Hill, R. 1965 Continuum micro-mechanics of elastoplastic polycrystals. J. Mech. Phys. Solids 13, 89-101. (doi:10.1016/0022-5096(65)90023-2)

Hutchinson, J. W. 1976 Bounds and self-consistent estimates for creep of polycrystalline materials. Proc. R. Soc. A 348, 101-127.

Kohn, R. V. \& Little, T. D. 1998 Some model problems of polycrystal plasticity with deficient basic crystals. SIAM J. Appl. Math. 59, 172-197. (doi:10.1137/S0036139997320019)

Milton, G. W. 2002 The theory of composites. Cambridge, UK: Cambridge University Press.

Nesi, V., Smyshlyaev, V. P. \& Willis, J. R. 2000 Improved bounds for the yield stress of a model polycrystalline material. J. Mech. Phys. Solids 48, 1799-1825. (doi:10.1016/S0022-5096(99)00100-3)

Olson, T. 1994 Improvements on Taylor upper bound for rigid-plastic composites. Mater. Sci. Eng. A 175, 15-20. (doi:10.1016/0921-5093(94)91039-1)

Parton, V. Z. \& Buryachenko, V. A. 1990 Stress fluctuations in elastic composites. Sov. Phys. Dokl. 35, 191-193. 
Ponte Castañeda, P. 1991 The effective mechanical properties of nonlinear isotropic composites. J. Mech. Phys. Solids 39, 45-71. (doi:10.1016/0022-5096(91)90030-R)

Ponte Castañeda, P. 1992 New variational principles in plasticity and their application to composite materials. J. Mech. Phys. Solids 40, 1757-1788. (doi:10.1016/0022-5096(92)90050-C)

Ponte Castañeda, P. \& Suquet, P. 1998 Nonlinear composites. Adv. Appl. Mech. 34, 171-302.

Rockafellar, T. 1970 Convex analysis. Princeton, NJ: Princeton University Press.

Suquet, P. 1993 Overall potentials and extremal surfaces of power law or ideally plastic materials. J. Mech. Phys. Solids 41, 981-1002. (doi:10.1016/0022-5096(93)90051-G)

Suquet, P. 1995 Overall properties of nonlinear composites: a modified secant moduli theory and its link with Ponte Castañeda's nonlinear variational procedure. C. R. Acad. Sci. Paris II 320, $563-571$.

Talbot, D. R. S. \& Willis, J. R. 1985 Variational principles for inhomogeneous nonlinear media. IMA J. Appl. Math. 35, 39-54.

Talbot, D. R. S. \& Willis, J. R. 1992 Some explicit bounds for the overall behavior of nonlinear composites. Int. J. Solids Struct. 29, 1981-1987. (doi:10.1016/0020-7683(92)90188-Y)

Willis, J. R. 1983 The overall response of composite materials. ASME J. Appl. Mech. 50, $1202-1209$.

Willis, J. R. 1992 On methods for bounding the overall properties of nonlinear composites: correction and addition. J. Mech. Phys. Solids 40, 441-445. (doi:10.1016/S0022-5096(05)80020-1)

Willis, J. R. 1994 Upper and lower bounds for nonlinear composite behavior. Mater. Sci. Eng. A 175, 7-14. (doi:10.1016/0921-5093(94)91038-3) 